\title{
A New Approach of Waveform Interpretation Applied in Nondestructive Testing of Defects in Rock Bolts Based on Mode Identification
}

\author{
Xiaoyang Rong, ${ }^{1}$ Peiyuan Lin, ${ }^{2}$ Jinyuan Liu, ${ }^{2}$ and Tianhong Yang ${ }^{1}$ \\ ${ }^{1}$ College of Resources \& Civil Engineering, Northeastern University, Shenyang 110819, China \\ ${ }^{2}$ Department of Civil Engineering, Ryerson University, Toronto, ON, Canada
}

Correspondence should be addressed to Tianhong Yang; yangtianhong@mail.neu.edu.cn

Received 7 December 2016; Accepted 9 February 2017; Published 5 March 2017

Academic Editor: Mitsuhiro Okayasu

Copyright (C) 2017 Xiaoyang Rong et al. This is an open access article distributed under the Creative Commons Attribution License, which permits unrestricted use, distribution, and reproduction in any medium, provided the original work is properly cited.

\begin{abstract}
Due to the characteristics of dispersion of guided waves, the waveforms recorded in ultrasonic nondestructive testing (NDT) of rock bolts are complicated to interpret. With a goal to increase the inspection sensitivity and accuracy in NDT of rock bolts, an approach of waveform interpretation based on wave modes identification is developed. The numerical simulation of full rock bolt and rock bolts with grout defect by Finite Element Method (FEM) is applied to illustrate the approach; it is found that the sensitive and low attenuation wave modes exist. Laboratory tests on full rock bolt and rock bolt with grout loss using NDT are conducted to evaluate the efficacy of the approach of waveform interpretation. In addition to that, a parametric study was conducted on rock bolt models with different sectional defect size. Based on the waveform interpretation, the mode-based reflection coefficient $R$ is proposed to evaluate the sensitivity of wave modes to the defect size of sectional area. It is found that the sensitivity of the wave mode does not change with the defect sectional area, and the amplitude depends on the size of the defect.
\end{abstract}

\section{Introduction}

Nowadays, rock bolts are used extensively to reinforce existing slopes or support deep excavations. The pullout capacity of a rock bolt is developed through the bonding between the grout column and the surrounding soil. An appropriately developed pullout resistance is the most critical factor for the success of a rock bolted structure. Many difficulties might arise during the grouting process in the field, such as necking due to borehole collapse, voids or defects in the grout column, or misalignment of the nails. The traditional method to examine the grouting quality for a rock bolt is to perform a pullout test, through which the integrity of the rock bolt system can be carefully checked. Although field testing is straightforward and reliable, it is expensive (i.e., cost of the bolts and testing equipment and time-consuming) and destructive. Thus, nondestructive testing (NDT) techniques which are less time-consuming, economic, and safe and allow the wave to travel a long distance with little loss in energy have been developed, which present bright prospects to overcome the above shortages of field testing.
Many NDT techniques have been widely applied for quality control or health condition assessment of infrastructure [1]. For rock bolts, there are few attempts recently to use different NDT techniques for nail integrity inspection, such as pulse echo method [2,3], electromagnetic induction method, and time domain reflectometry method [4]. These studies demonstrated the advantages of NDT for nail grouting quality evaluation (e.g., cost-effective, time-efficient, and nondestructive). In addition to these methods, an alternative NDT technique for rock bolt grout quality inspection can be developed based on the ultrasonic guided wave theory. The ultrasonic guided wave theory has been applied to study the integrity of rock bolts [5-9] and concrete piles [10]. The ultrasonic guided wave theory is adopted in this study for the grouting quality inspection of rock bolts. A wave would travel back by reflection along a rock bolt once it has reached the end of the bolt, during which the consecutive waveforms recorded at one location on a bolt can be used to analyze the characteristics of wave propagation [8]. Therefore, the method to identify the wave echo is crucial to the accuracy of analysis. 
The group velocity, which is the apparent propagation speed of wave energy if there is no energy leak, is the wave velocity that can be measured in experiments. A considerable amount of literature $[8,11,12]$ also confirmed the dependency of the group velocity of guided wave on material properties and wave frequency. However, in ultrasonic guided waves, there are numerous modes [13]. Due to wave dispersion, the wave velocity varies with the elastic properties and density of the medium, as well as the frequency, the geometry, and the wave mode. For a certain frequency and geometry, several modes may propagate with different velocities. In general, an excitation source can excite all modes of waves within a certain frequency bandwidth, resulting in dispersive deformation of waveform which is too complicated to interpret.

So it is desired to provide a solution to identify the group velocity and traveling time. Zou et al. [8] used the average amplitude over a time interval to calculate the amplitude ratio to identify the complicated echoes especially when the bolt is short or when the dispersion is serious. This method makes it easier in practice to estimate the energy, especially when the boundary between the first arrival and the echo in grouted rock bolts is difficult to identify because of dispersion. Because the amplitude ratio determined by this method varies with the length of the time interval, the accuracy of the average amplitude method remains to be improved. Additionally, great care is needed for the correct identification of reflections from defects and normal rock bolt end. So our research characterized the behavior of each group of wave echo to the exact mode to analyze the guided waves propagation in rock bolt, which increased the accuracy of inspection substantially.

Certain wave mode has been used as excitation wave by a number of authors to investigate the inspection sensitivity of steel embedded in concrete, which has proved the feasibility and validity. In a recent publication on corroded steel bars embedded in concrete, Ervin and Reis [14] and Ervin et al. [15] used both low $[<200 \mathrm{kHz}, L(0,1)$ mode] and high $[2-9 \mathrm{MHz}, L(0,9)$ mode] guided longitudinal modes to ultrasonically monitor the accelerated corrosion. Sharma and Mukherjee [16] found that some modes were sensitive to the combined effect of bond deterioration and mortar stiffness reduction, while some other modes correlated well with the change in cross-sectional area. It was confirmed by Sharma and Mukherjee [17] that specific core and surface seeking modes of ultrasonic guided waves were successfully used to identify the type, rate, and mechanism of corrosion in a reinforcing bar in concrete subjected to different exposure conditions. Generally, these modes have attenuation minima, or dips, with minimal amounts of wave energy lost due to leakage and material absorption [17]. So it can be induced that mode being sensitive to the loss of wave energy due to defect in the steel-grout system can be distinguished, which will be feasible and of great benefit to improve the accuracy.

This study presents an approach to investigate the behavior of wave propagation in detection of rock bolts by sensitive modes identification. Dispersion curves were given by the derivation of longitudinal wave equations to provide group velocities and modes against the input frequencies of guided waves in rock bolts. A full rock bolt and nine rock bolts

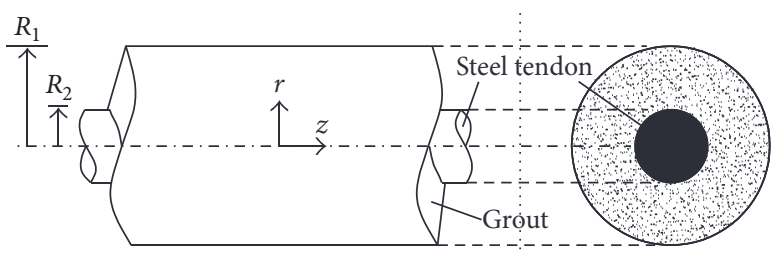

FIGURE 1: A tendon-grout composite system for rock bolt with cylindrical coordinate system.

with different size of defect in grout were simulated. At first, behavior of wave propagation in a full rock bolt was compared with a defective rock. The approach of interpretation of waveforms based on mode identification is then presented and the sensitive mode is given. Laboratory tests on rock bolts using NDT are conducted to evaluate the efficacy of the approach of waveform interpretation. In addition to that, the parametric study on rock bolts with different sectional size is conducted. It is found that the sensitivity of the wave mode does not change with the defect sectional area, which also confirmed the accuracy of the method.

\section{Dispersion Curves of Longitudinal Guided Waves for the Tendon-Grout Composite System}

The theoretical wave model for rock bolts is briefly introduced in this section. An idealized grouted rock bolt can be simplified as a tendon-grout composite system, as shown in Figure 1. The inner layer is the steel tendon with a radius of $R_{2}$, while the outer layer is a grout hollow bar with a thickness of $\left(R_{1}-R_{2}\right)$.

Introducing two dynamic potential functions, $\Phi$ and $\psi$, the longitudinal wave equations can be expressed using the cylindrical coordinates $r, \theta$, and $z$ as [18]

$$
\begin{aligned}
& \nabla^{2} \Phi=\frac{1}{c_{L}^{2}} \frac{\partial^{2} \Phi}{\partial t^{2}}, \\
& \nabla^{2} \Psi=\frac{1}{c_{T}^{2}} \frac{\partial^{2} \Psi}{\partial t^{2}}
\end{aligned}
$$

where $c_{L}^{2}=(\lambda+2 \mu) / \rho$ and $c_{T}^{2}=\mu / \rho$ are the dilatational and shear wave velocity, respectively; $\lambda$ and $\mu$ are the first and second Lame constants, respectively; $\rho$ is the density of the material; and $\nabla^{2}=\partial^{2} / \partial r^{2}+(1 / r)(\partial / \partial r)+\partial^{2} / \partial z^{2}$.

For longitudinal waves, the displacement $u_{\theta}$ vanishes because of the symmetry. Only the radial and axial displacements, $u_{r}$ and $u_{z}$, are considered in the nail. The two displacement components can be calculated by the following two potentials:

$$
\begin{aligned}
& u_{r}=\frac{\partial \Phi}{\partial r}+\frac{\partial^{2} \Psi}{\partial r \partial z} \\
& u_{z}=\frac{\partial \Phi}{\partial z}-\frac{\partial^{2} \Psi}{\partial r^{2}}-\frac{1}{r} \frac{\partial \Psi}{\partial r}
\end{aligned}
$$


The stresses caused by the propagation of longitudinal waves can be calculated through Hooke's law as

$$
\begin{aligned}
\sigma_{r r} & =2 \mu \frac{\partial u_{r}}{\partial r}+\lambda\left(\frac{u_{r}}{r}+\frac{\partial u_{r}}{\partial r}+\frac{\partial u_{z}}{\partial z}\right), \\
\sigma_{r z} & =\mu\left(\frac{\partial u_{r}}{\partial z}+\frac{\partial u_{z}}{\partial r}\right),
\end{aligned}
$$

where $\sigma_{r r}$ and $\sigma_{r z}$ are the stresses in $r$ and $z$ directions, respectively.

Provided that the harmonic waves propagate along the $z$ axis, the general solutions for (1a) and (1b) are derived:

$\Phi$

$$
= \begin{cases}{\left[A_{1} J_{0}\left(\alpha_{1} r_{1}\right)+B_{1} Y_{0}\left(\alpha_{1} r_{1}\right)\right] e^{i(k z-\omega t)},} & R_{2} \leq r_{1} \leq R_{1}, \\ A_{2} J_{0}\left(\alpha_{2} r_{2}\right) e^{i(k z-\omega t)}, & 0 \leq r_{2} \leq R_{2},\end{cases}
$$

$\Psi$

$$
= \begin{cases}{\left[C_{1} J_{0}\left(\beta_{1} r_{1}\right)+D_{1} Y_{0}\left(\beta_{1} r_{1}\right)\right] e^{i(k z-\omega t)},} & R_{2} \leq r_{1} \leq R_{1}, \\ C_{2} J_{0}\left(\beta_{2} r_{2}\right) e^{i(k z-\omega t)}, & 0 \leq r_{2} \leq R_{2},\end{cases}
$$

where $k$ is the wave number that can be calculated as $k=$ $\omega / c_{p} ; c_{p}$ is the phase velocity; $\omega$ is the circular frequency defined as $\omega=2 \pi f$ (i.e., $f$ is the frequency); $t$ is the travel time of the wave; and $i$ is the imaginary unit. Parameters $A_{1}, B_{1}$, $C_{1}, D_{1}, A_{2}$, and $C_{2}$ are unknown constants; $\alpha^{2}=\omega^{2} / c_{L}^{2}-k^{2}$; $\beta^{2}=\omega^{2} / c_{T}^{2}-k^{2} ; J_{0}$ is the Bessel functions of the first kind; and $Y_{0}$ is the Bessel functions of the second kind.

In total, there are six unknown constants to be determined, that is, $C=\left[A_{1}, B_{1}, C_{1}, D_{1}, A_{2}, C_{2}\right]$. Because the stresses and strains induced by the propagation of longitudinal waves are very small, both the steel and the grout work in the linear elastic range. Moreover, these two layers are usually assumed to be perfectly bonded without relative movement at the interface. Therefore, a hard contact at the tendon-grout interface is appropriate, so that both the displacements and stresses at the steel tendon and the grout should be compatible at $r_{1}=r_{2}=R_{2}$. In addition, at the nail surface, that is, $r_{1}=R_{1}$, the stresses must be zero when no external force is applied. Hence, we obtain

$$
\mathbf{M C}=0
$$

where $\mathbf{M}$ is a $6 \times 6$ matrix, and the expression of each element is given in appendix. The determinant of the matrix $\mathbf{M}$ must be zero to have nontrivial solutions, yielding

$$
F\left(c_{p}, f\right)=\operatorname{det}[\mathbf{M}]=0 .
$$

Equation (6) can be plotted to produce the dispersion curves for a rock bolt. For a specific wave mode, once the frequency of excitation is known, the phase velocity can be evaluated from the dispersion curves. The unknown matrix of $\mathrm{C}$ can then be solved by substituting a prefixed frequency and the corresponding phase velocity into (5). Once the constants $\mathbf{C}$ are determined, the displacements and stresses induced by the specific mode in the rock bolt can be calculated. The displacements and stresses obtained from each specific wave mode are superposed to calculate the total displacements and stresses.

The physical properties of the grout-bolt system for use in the calculation include the values of density, $\rho$, Young's modulus, $E$, and Poisson's ratio, $v$. Other parameters, such as Lame's constants and wave velocities, were derived from three basic variables. Table 1 summarizes the values used to develop the dispersion curves.

Longitudinal waveforms have axial and radial displacements that are represented by the notation $L(m, n)$. The characters " $m$ " and " $n$ " represent the circumferential displacement (function of $\cos (m \theta)$ ) and the sequential order of the mode, respectively [14]. As longitudinal waves are employed in this investigation, only the axially symmetric (order 0) modes are excited. A simulated scaled rock bolt model has been used to illustrate how the developed method can be applied in the analysis of longitudinal wave propagation. The rock bolt has an overall length of $615 \mathrm{~mm}$, an outer grout thickness of $25 \mathrm{~mm}$, and inner steel radius of $5 \mathrm{~mm}$. Based on (6) and the parameters given in Table 1, the dispersion curves of group velocity and phase velocity in the frequency range of 0 to $200 \mathrm{kHz}$ calculated according to the model and material parameters are shown in Figure 2 for the first sixorder modes. As can be seen from Figure 2, the lower the frequency, the lower the dispersion.

The phase velocities and group velocities are inconsistent; the stronger the dispersion, the bigger the difference of them. It can be seen that the phase velocities overall showed a downward trend directly, while the group velocities fluctuate greatly.

The individual harmonics travel with different phased velocities $c_{p}$, but the superimposed packet travels with the group velocity $c_{g}$. Realistically, we should therefore consider the velocity of a superposition of a number of waves, rather than the individuals [11]. The velocity of wave packets for a finite wave train can be interpreted from the group velocity dispersion curves; they are therefore useful to calculate the travel times of wave signals for use in the long-range testing [19]. For bars embedded in grout, leakage plays an important role. For long-range guided wave inspection, the low frequency range is of primary interest from a wave attenuation point of view. The frequency of $100 \mathrm{kHz}$ is therefore selected in this paper. The theoretical values of group velocities of the first six-order longitudinal modes are determined using Figure 2, as shown in Table 2.

\section{A New Approach of Waveform Interpretation Based on Sensitive Modes Identification}

3.1. FEM Model for Wave Propagation Simulation. The finite element package, ABAQUS/Explicit, is employed to simulate wave propagation along a rock bolt. Modeling a full-scale rock bolt involves a huge number of elements, which will cause computational difficulties in terms of calculation time, computer hardware, and storage issues. Thus, a scaled rock 
TABLE 1: Material properties used to develop the dispersion curves.

\begin{tabular}{|c|c|c|c|c|c|c|c|}
\hline \multirow{3}{*}{ Material } & \multicolumn{3}{|c|}{ Basic variables } & \multicolumn{4}{|c|}{ Derived variables } \\
\hline & \multirow{2}{*}{ Density, $\rho\left(\mathrm{kg} / \mathrm{m}^{3}\right)$} & \multirow{2}{*}{ Young's modulus, $E(\mathrm{GPa})$} & \multirow{2}{*}{ Poisson's ratio, $v$} & \multicolumn{2}{|c|}{ Lame's constants } & \multirow{2}{*}{$c_{L}(\mathrm{~km} / \mathrm{s})$} & \multirow{2}{*}{$c_{T}(\mathrm{~km} / \mathrm{s})$} \\
\hline & & & & $\mu(\mathrm{GPa})$ & $\lambda(\mathrm{GPa})$ & & \\
\hline Steel & 7840 & 210 & 0.30 & 121.15 & 80.77 & 6.00 & 3.21 \\
\hline Grout & 2400 & 17 & 0.20 & 4.72 & 7.08 & 2.80 & 1.72 \\
\hline
\end{tabular}

TABLE 2: Group velocities for the first six-order longitudinal modes at $100 \mathrm{kHz}$.

\begin{tabular}{lcccccc}
\hline Mode & $L(0,1)$ & $L(0,2)$ & $L(0,3)$ & $L(0,4)$ & $L(0,5)$ & $L(0,6)$ \\
\hline Group velocity, $c_{g}(\mathrm{~km} / \mathrm{s})$ & 1.75 & 1.35 & 2.40 & 1.80 & 2.60 & 1.25 \\
\hline
\end{tabular}

bolt model with an overall length of $615 \mathrm{~mm}$, a grout thickness of $25 \mathrm{~mm}$, and steel radial of $5 \mathrm{~mm}$ is simulated in this study. A solid element type, C3D8R (an 8-node linear brick, reduced integration, and hourglass control), is selected for the steel rod and grout. It is found that element size should be sufficiently small, normally in the range from $1 / 10$ to $1 / 20$ of the minimum wave length $\left(\lambda_{s}\right)$, to capture the wave response $[20,21]$. Given the frequency of $100 \mathrm{kHz}$ and the dilatational velocity of $C_{T}=6000 \mathrm{~m} / \mathrm{s}$ for steel (Table 1 ), the element size is calculated as $L_{e}=\lambda_{s} / 10=C_{T} / 20 \pi f=1 \mathrm{~mm}$. There are a total of 477171 nodes and 467400 elements in the numerical model. For the processing of boundary conditions, rigid boundaries are set at the end of the steel tendon [22]. The input parameters of the model are listed in Table 3 , which is the same as that used by Cui and Zou [6].

The excitation can be generated using a narrowband windowed sinusoidal signal as the time-dependent amplitude of pressure. The windowed signal with ten cycles is used, having an input frequency of $100 \mathrm{kHz}$ and duration of $1 \times$ $10^{-4} \mathrm{~s}$, as shown in Figure 3. The incident wave with a magnitude of $10 \mathrm{MPa}$ is applied perpendicular to the cross section of the steel bar.

A total of 10 rock bolt models with different sectional defects are applied to study the effect of defect on the wave propagation in this paper. The reflection occurred due to the sudden change of acoustic impedance at the defect surface; especially when the wave propagates from high acoustic impedance medium into low acoustic impedance, the area of the defect surface will affect the wave propagation greatly. While when the wave propagates from low acoustic impedance medium into high acoustic impedance, the wave propagates along the medium with little reflection. So the grout losses with different cross-sectional area are of great interest, while the width of the defect along the rock bolt is chosen as a constant value $(33 \mathrm{~mm})$. Moreover, the defect is located in the middle ( $308 \mathrm{~mm}$ far from the incident plane) along the rock bolt to get a clear boundary to distinguish the echoes of the defect and the end of the rock bolt because of dispersion. Different grout losses with different crosssectional area (reduced by $0,21.45 \%, 33.36 \%, 40.51 \%, 50.04 \%$, $64.34 \%, 71.48 \%, 78.63 \%, 85.78 \%$, and $95.31 \%$ ) in the grout are applied to simulate the grout defects. Figure 4 presents the mesh discretization of the finite element model for the rock bolt with a $50.4 \%$ sectional defect.
3.2. Studies of Behavior of Wave Propagation in Rock Bolts. A waveguide is a physical structure which supports the propagation of mechanical waves along its elongated direction and modifies the behavior of waves by discretizing waves into propagating modes and relating wave number and frequency in a nonlinear way. As a consequence, signals with a significant bandwidth are distorted as they travel along the waveguide [23]. The reason for that is the group velocity difference between different modes of a certain frequency, the wave groups of different modes separate from each other as the time increases. The longer the propagating time, the more obvious the separation of the wave group is induced by dispersion. The process of waveform deformation induced by separation of wave group of different modes is visually displayed in the modeling results of displacement magnitude $U$ for a fully grouted rock bolt and a rock bolt with a $50.4 \%$ sectional defect shown in Figures 5 and 6, respectively.

Figure 5(a) shows the displacement induced by the incident wave at $0.1 \mathrm{~ms}$, which remains a wave group broadly. Comparing Figures 5(b) and 5(c) with 5(a), there are mainly two separated wave groups propagating along the rock bolt, and the distance increased as the time increased. Figure 5(c) shows that the first wave group reached the end of the rock bolt at $0.3 \mathrm{~ms}$. The first wave echo met the second wave group at $0.4 \mathrm{~ms}$ as shown in Figure 6(d); the displacement of each node is the sum of the displacement induced by the two wave groups. As can be seen in Figure 5(e), there are three distinct propagating wave groups at $0.5 \mathrm{~ms}$. The first wave group propagates mainly in the steel tendon along the longitudinal direction with little energy leaked into the grout. The second and third wave groups display both the longitudinal and shear behavior, propagating in both the steel and the grout.

Comparing with a fully grouted rock bolt in Figure 5, Figure 6 demonstrates that loss of grout altered the transient behavior and distributions of guided waves for a rock bolt model with a defect. As shown in Figure 6(a), the behavior of the guided wave is exactly the same as the fully grouted rock bolts (see Figure 5(a)), because the incident wave has not reached the defect. By the time of $0.2 \mathrm{~ms}$ as in Figure 6(b), significant wave reflection occurs due to the sudden change of acoustic impedance at the defect surface, so the reflected waves are not axisymmetric any more. The defect echoes reach the loading surface at $0.4 \mathrm{~ms}$, which is highlighted using a red circle as shown in Figure 6(d). The groups of wave 
TABLE 3: Material parameters used in simulation.

\begin{tabular}{lccc}
\hline Material & Density, $\rho\left(\mathrm{kg} / \mathrm{m}^{3}\right)$ & Young's modulus, $E(\mathrm{GPa})$ & Poisson's ratio, $v$ \\
\hline Steel & 7840 & 210 & 0.30 \\
Grout & 2400 & 17 & 0.20 \\
\hline
\end{tabular}
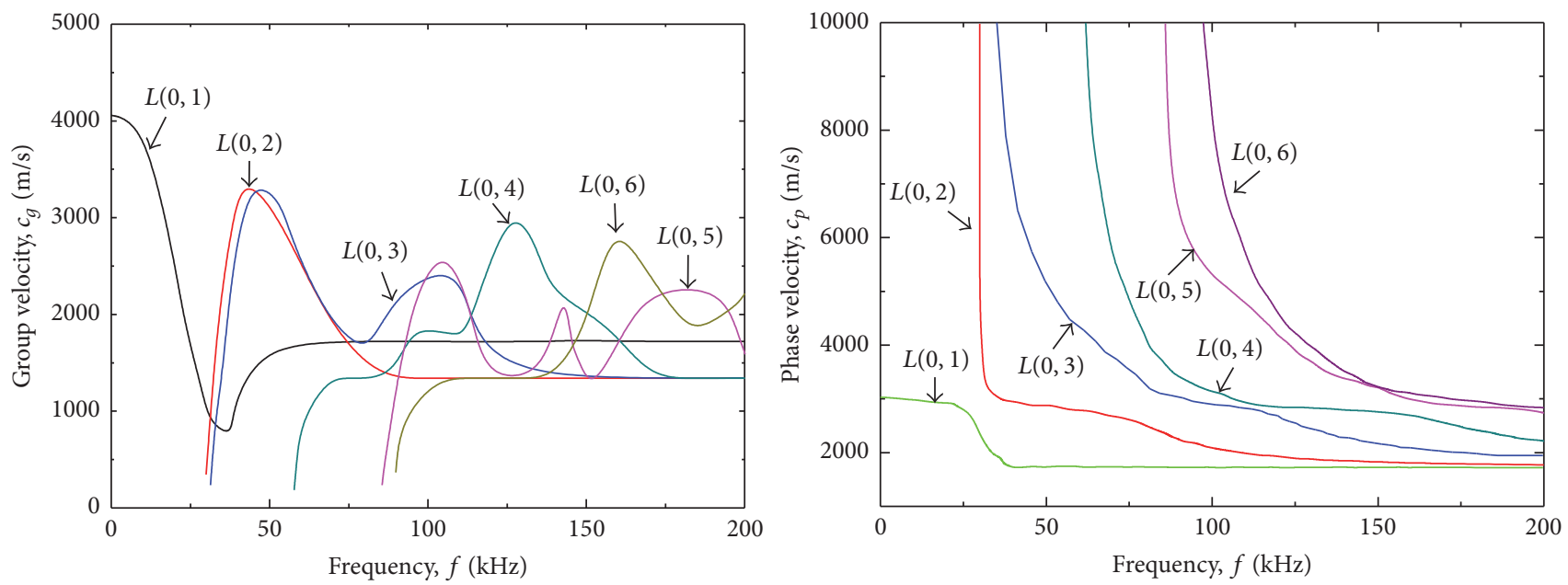

FIGURE 2: Group velocity and phase velocity of the first six $L(0, m)$ modes of the for the steel tendon-grout system.

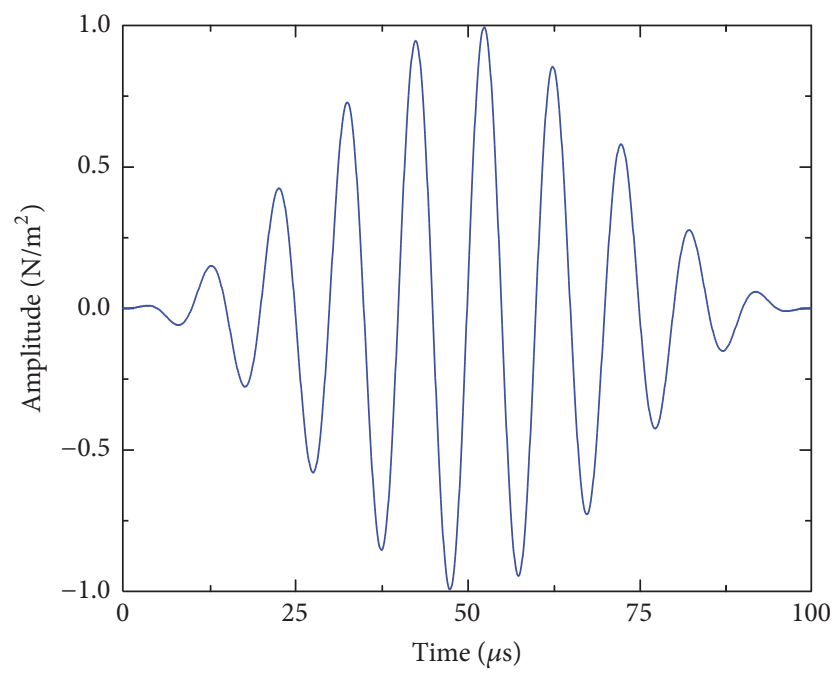

FIgURE 3: Excitation signal (100 kHz).

modes are separated from each other over time, as shown in Figure 6(e).

\subsection{Interpretation of Waveforms in NDT of Rock Bolts Based} on Sensitive Modes Identification. The guided waves are dispersive, where the groups of wave modes are dependent on their properties such as velocity and attenuation. The distorted waveforms induced by dispersion create difficulties in the interpretation of signals. There are an infinite number of wave modes propagating in the rock bolt, and each mode has the corresponding velocity and attenuation. Zou et al. attributed attenuation to three sources: (a) energy may be dissipated during wave propagation in a medium due to nonelastic resistance; (b) energy loss occurs when deformation of waveform due to dispersion occurs; (c) energy is spread at the interface between the bolt and the grout [8]. Two frequency-dependent attenuation components (i.e., dispersive and spreading attenuation) were considered to evaluate the amplitude ratio as an indirect measurement of attenuation using the average amplitude defined in (7). The average amplitude over a time interval was used to calculate the amplitude ratio to identify the complicated echoes especially when the bolt is short or when the dispersion is serious. The equation has the following form [8]:

$$
\begin{array}{r}
A_{i}=\sqrt{\frac{\int_{t_{i 1}}^{t_{i 2}} k v_{i}^{2}(t) d t}{(1 / 2) k\left(t_{i 2}-t_{i 1}\right)}}=\sqrt{\frac{2}{t_{i 2}-t_{i 1}} \int_{t_{i 1}}^{t_{i 2}} v_{i}^{2}(t) d t} \\
i=1,2 .
\end{array}
$$

In this expression, $\Delta t_{i}=t_{i 2}-t_{i 1}$ is the time interval centered at the maximum amplitude of wave packet, $v_{i}(t)$ is the recorded wave amplitude, $i=1$ indicates the first arrival, $i=2$ corresponds to the echo, and $k$ is a material constant.

This method makes it easier in practice to estimate the energy, especially when the boundary between the first arrival and the echo in grouted rock bolts is difficult to identify because of dispersion. Because the amplitude ratio determined by this method varies with the length of the time interval, the accuracy of the average amplitude method remains to be improved. Additionally, great care is needed for the correct identification of reflections from defects and normal rock bolt end. The approach of interpreting waveforms by characterizing each group of wave echo to the exact mode to analyze the guided waves propagation 


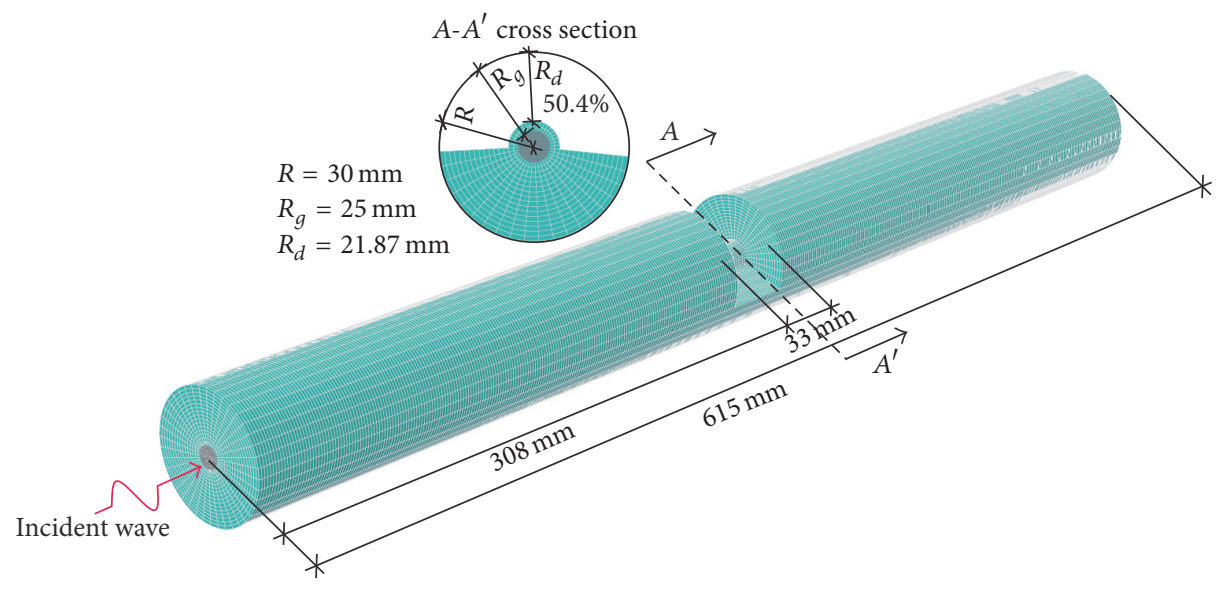

FIgURE 4: Finite element model of rock bolt with a $50.4 \%$ sectional grout defect.

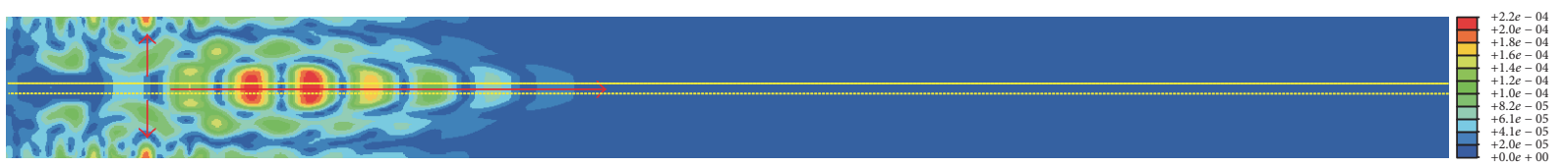

(a)

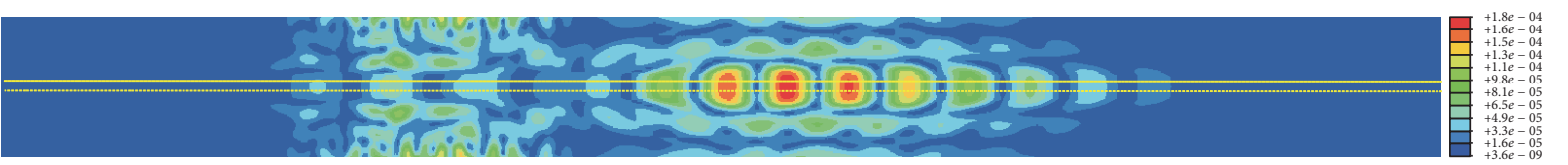

(b)

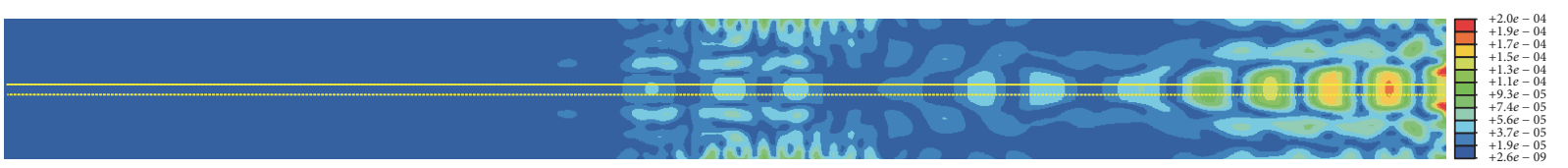

(c)

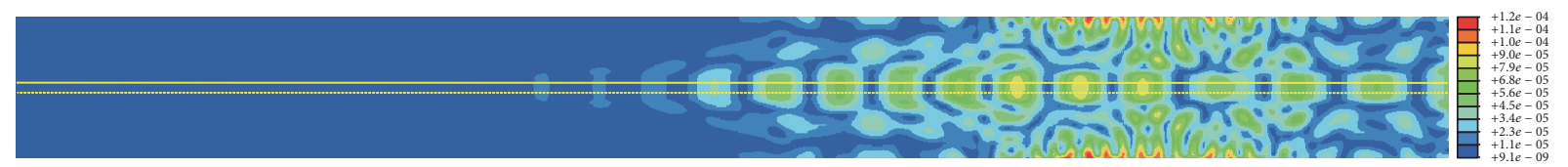

(d)

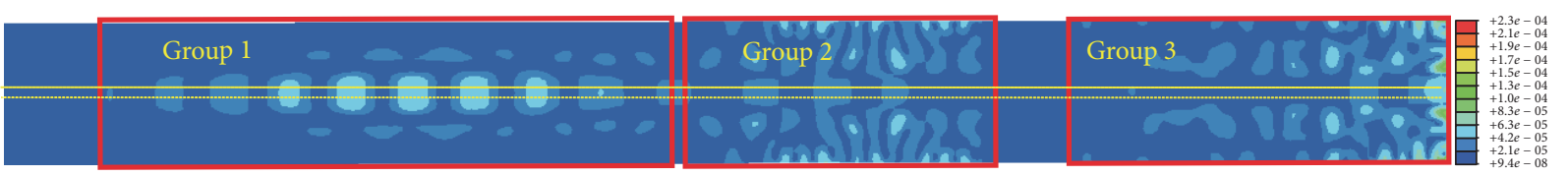

(e)

FiguRE 5: Cross-sectional magnitude displacement $U$ of the wave propagation at $100 \mathrm{kHz}$ along a fully grouted rock bolt at (a) time $=0.1 \mathrm{~ms}$, (b) time $=0.2 \mathrm{~ms}$, (c) time $=0.3 \mathrm{~ms}$, (d) time $=0.4 \mathrm{~ms}$, and (e) time $=0.5 \mathrm{~ms}$.

aimed at increasing the accuracy of inspection of rock bolt substantially is proposed. The analysis below is taken as a case to demonstrate the approach.

The waveforms of nodal displacement on the loading surface for the fully grouted rock bolt and the rock bolt with a $50.4 \%$ defect are shown in Figure 7 . The velocities of the first six modes excited at $100 \mathrm{kHz}$ and the behavior of guided waves as given in Figures 5 and 6 are combined to guide the interpretation the waveforms.
The waveforms at the time interval between 0 and $0.1 \mathrm{~ms}$ in Figure 10 correspond to Figure 5(a), which are induced by the incident wave. From $0.1 \mathrm{~ms}$ to $0.5 \mathrm{~ms}$, the guided waves transmit through the rock bolt. From approximately $0.5 \mathrm{~ms}$, three separate groups of waves arrive in turn, as shown in Figure 7(a), which are corresponding to the three wave groups in Figure 5(e). The velocity of the first wave group, denoted as $V_{g 1}$, is calculated as $V_{g 1}=2 \times 0.615 \mathrm{~m} / 5.19 \mathrm{~ms}=$ $2369.94 \mathrm{~m} / \mathrm{s}$. The duration of the wave echo $(5.19 \mathrm{~ms})$ is 


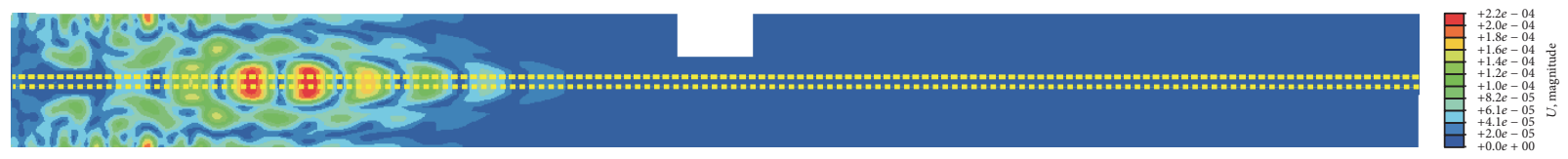

(a)

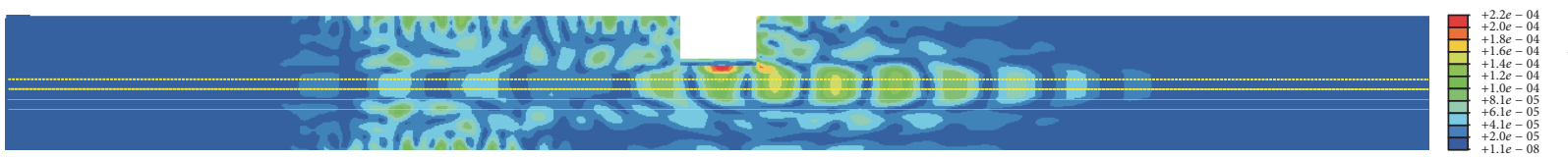

(b)

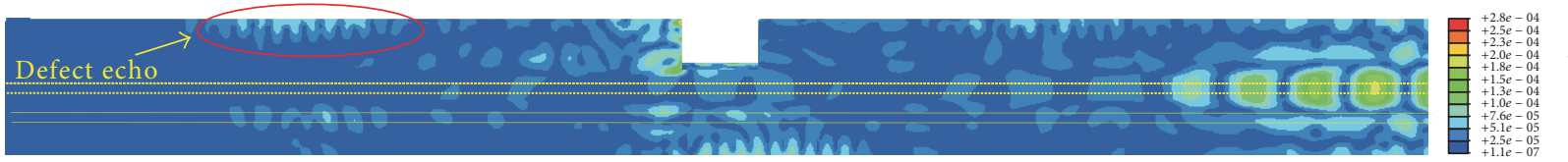

(c)

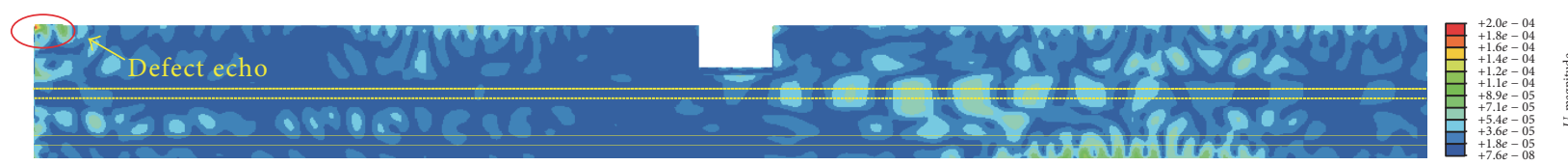

(d)

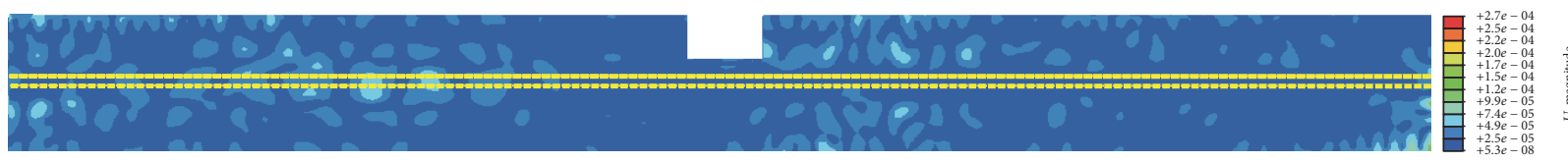

(e)

FIGURE 6: Wave propagation modeling results of displacement magnitude $U$, at $100 \mathrm{kHz}$ along a rock bolt with a defect at $(\mathrm{a})$ time $=0.1 \mathrm{~ms}$, (b) time $=0.2 \mathrm{~ms}$, (c) time $=0.3 \mathrm{~ms}$, (d) time $=0.4 \mathrm{~ms}$, and (e) time $=0.5 \mathrm{~ms}$.

the time difference between the peak-to-peak value of the incident wave and the first wave echo. The calculation agrees well with the theoretical group velocity of $2400 \mathrm{~km} / \mathrm{s}$ for the mode $L(0,3)$ as tabulated in Table 2 .

The velocity of the second wave group, denoted as $V_{92}$, is calculated as $V_{g 2}=2 \times 0.615 \mathrm{~m} / 0.65 \mathrm{~ms}=1892 \mathrm{~m} / \mathrm{s}$. The duration of the wave echo $(0.65 \mathrm{~ms})$ is estimated using the time difference between the peak-to-peak value of the incident wave and the second wave echo. Again, a reasonable match between numerical calculation and theoretical derivation (i.e., $1800 \mathrm{~m} / \mathrm{s}$ for the mode $L(0,4)$, Table 2 ) is obtained.

The velocity of the third wave group, calculated as $V_{g 3}=$ $2 \times 0.615 \mathrm{~m} / 0.707 \mathrm{~ms}=1739.74 \mathrm{~m} / \mathrm{s}$, is consistent with the theoretical velocity of $1750 \mathrm{~m} / \mathrm{s}$ for the mode $L(0,1)$ as given in Table 2. The arriving time of the mode $L(0,2)$ is denoted as $T_{m 4}$, which is estimated as $T_{m 4}=2 \times 0.615 \mathrm{~m} / 1350 \mathrm{~m} / \mathrm{s}=$ $0.91 \mathrm{~ms}$. All these modes of wave groups are highlighted in Figure 7(a).

For the waveforms of the rock bolt with a defect, Figure 6(d) indicates that an obvious defect echo arrived at the loading surface at $0.4 \mathrm{~ms}$, which matches well with the defect echo at about $0.4 \mathrm{~ms}$ in Figure 7(b). A relatively smaller defect echo is recorded at $0.3 \mathrm{~ms}$ as shown in Figure 7(b), although it could not be seen in Figure 6(c).

Comparing the waveforms of rock bolts with and without defects, two more defect echoes are generated approximately at $0.5 \mathrm{~ms}$ and $0.9 \mathrm{~ms}$, respectively. As the defect echoes should be earlier than the echoes from the end of the rock bolt, the first and second defect echoes are suggested for use to determine the location of the defect.

For the tests in real sites, low attenuation and easily distinguished mode is considered. The amplitude of the 1st defect echo is $35.06 \%$ of the 2 nd defect echo, so the defect 2 is considered. The two defect echoes' velocities, denoted as $V_{\text {defect- } 1}$ and $V_{\text {defect-2 }}$, are calculated as $V_{\text {defect-1 }}=2 \times 0.308 \mathrm{~m} / 0.249 \mathrm{~ms}=$ $2470 \mathrm{~m} / \mathrm{s}$ and $V_{\text {defect-2 }}=2 \times 0.308 \mathrm{~m} / 0.362 \mathrm{~ms}=1740 \mathrm{~m} / \mathrm{s}$. They are comparable to the theoretical group velocity of $2400 \mathrm{~km} / \mathrm{s}$ for the mode $L(0,3)$ and of $1750 \mathrm{~m} / \mathrm{s}$ for the mode $L(0,1)$, respectively. Moreover, the third group of wave mode $L(0,1)$ propagates in both the steel and grout, while the first group of wave mode $L(0,3)$ propagates mainly in the steel bar. Therefore, it could be considered that the mode $L(0,1)$ was more sensitive to the loss of grout.

\section{Assessment of the Approach of Waveform Interpretation by Laboratory Tests}

Experimental data and theoretical derivation of velocity were applied to evaluate the efficacy of the approach of waveform interpretation.

4.1. Evaluation by Laboratory Test of Rock Bolts Using NDT. An ultrasonic guided wave NDT system is employed 


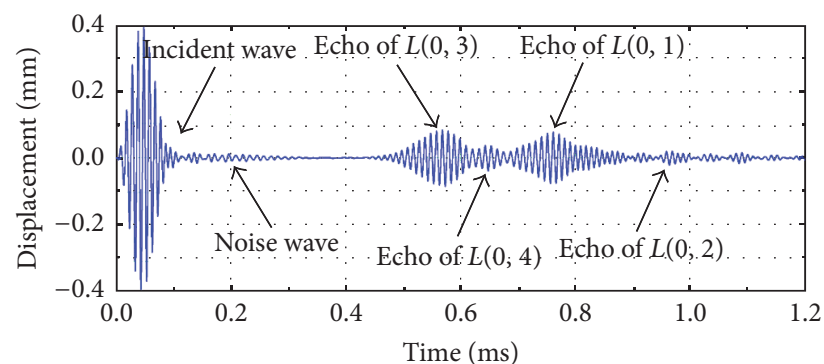

(a)

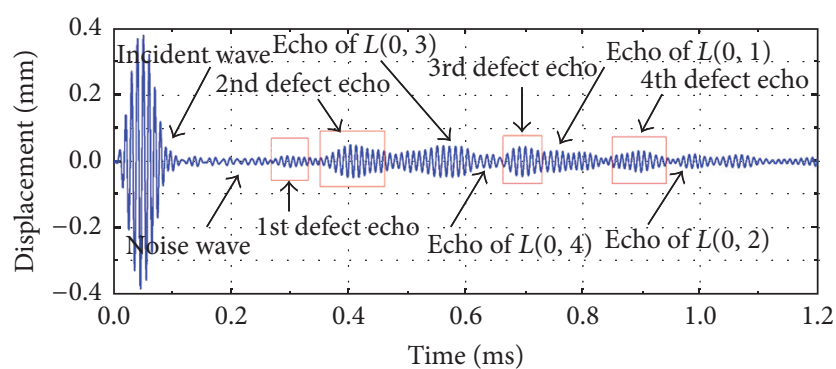

(b)

FIGURE 7: Waveforms of nodal displacement on the loading surface: (a) fully grouted rock bolt and (b) rock bolt with grout loss.

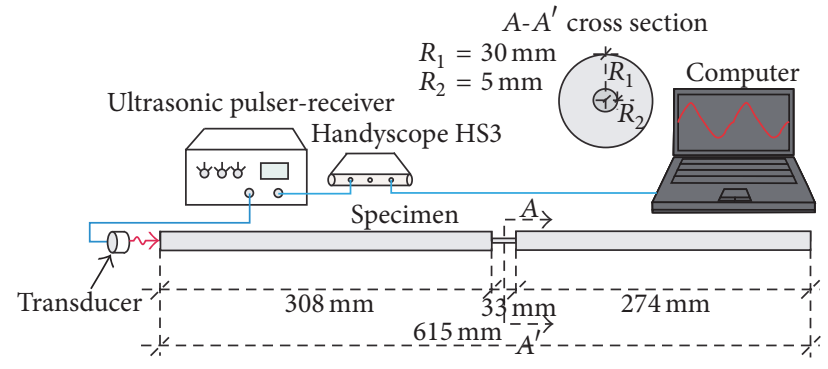

FIGURE 8: Illustration for through-transmission ultrasonic testing setup.

in this study, which consists of an ultrasonic pulserreceiver (5077PR, Square Wave Pulser/Receiver, Olympus), a Handyscope HS3, cables, and a PC. Narrowband transducer that sends a windowed toneburst at the central frequency of $100 \mathrm{kHz}$ is used in the tests. The transmitting transducer is self-transmitting and self-receiving (Contact Transducer, $100 \mathrm{KHz}, 39 \mathrm{~mm}$. Element Diameter). The transducer is gel coupled to the plane ends of the bar. Illustration for throughtransmission ultrasonic testing setup is shown in Figure 8.

The experiments are conducted on an intact grouted rock bolt and a rock bolt with grout loss to verify the approach of waveform interpretation. The intact grouted rock bolt is a $615 \mathrm{~mm}$ long and $10 \mathrm{~mm}$ diameter steel tendon filled with grout at the center of a plastic pipe. The pipe has an internal diameter of approximately $30 \mathrm{~mm}$. The grouted sections cover almost the whole bar, leaving section about $5 \mathrm{~mm}$ long protruding from the grout at each end for fixing of the bar. The rock bolt with grout loss is realized by putting a plastic foam material into the pipe when the grout is poured so they are not filled with grout. The specimens of full grouted rock bolt and rock bolt with grout loss are shown in Figure 9.

Both measured results of the two specimens along the rock bolts are normalized against the maximum deformations, as shown in Figures 10(a) and 10(b). The different modes of the main wave groups identified by the approach of waveform interpretation are identical with the numerical results. In general, the experimental waveforms are comparable to the calculated waveforms in rock bolts using numerical method.

The comparison of the velocities calculated by theoretical, numerical, and experimental method is shown in Table 4.

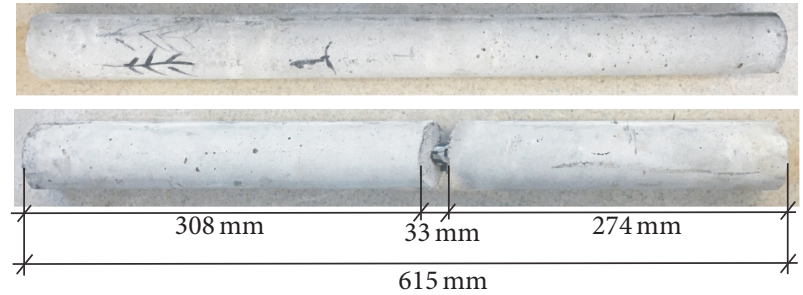

FIGURE 9: Full grouted rock bolt and rock bolt with grout loss specimens.

The errors between the end echo velocities of mode $L(0,1)$ in full rock bolt and rock bolt with grout loss calculated by the theoretical and experimental method are both $0.57 \%$. The errors between the end echo velocities of mode $L(0,1)$ in full rock bolt and rock bolt with grout loss calculated by the numerical and experimental method are $0.57 \%$ and $2.27 \%$, respectively, which provided confidence that the approach of waveform interpretation based on mode identification could be employed to study guided wave propagation in a rock bolt.

The small difference between FEM calculations and measured waveforms is attributed to two main factors. The first is the noise echoes induced by the rough surface of steel bar. In manual testing, coupling is usually achieved with a gel. However, inadequate coupling is a common cause of poor results, which could not be simulated explicitly in the numerical model. The second factor is the difference in input parameters, as an idealized wave may have filtered some high frequency low amplitude waves in the numerical model.

\section{Parametric Study on Sensitive Wave Mode for Determination of Defect Size}

Theoretically, when a wave reaches a medium which does not transmit mechanical waves (e.g., vacuum or air), no refraction occurs and all energy is reflected back from the defect surface [24]. For rock bolts, a defect will induce reflection of waves due to changes in acoustic impedances. So the size of the surface influences the defect echo significantly. A parametric study for rock bolt models with different sectional defects (i.e., cross section area reduced by $0,21.45 \%$, $26.21 \%, 30.98 \%$, 40.51\%, 50.04\%, 57.19\%, 64.34\%, 71.48\%, $78.63 \%, 85.78 \%$, and $95.31 \%)$ is conducted to analyze the 


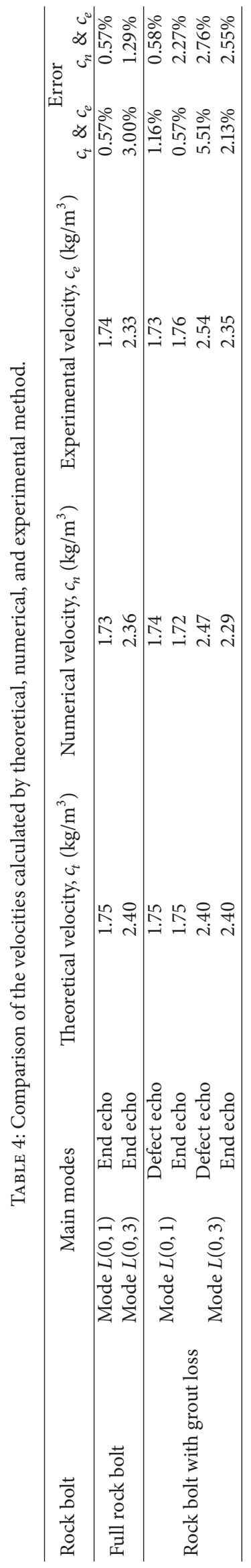




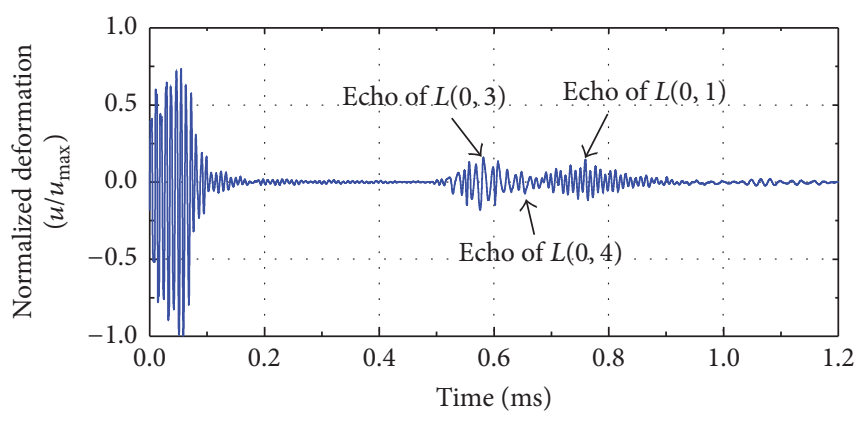

(a)

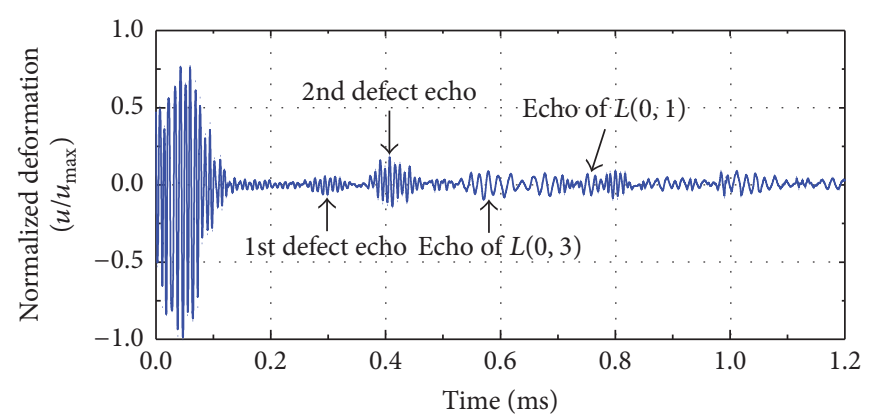

(b)

FIGURE 10: Typical measured ultrasonic waveforms at $100 \mathrm{kHz}$ in rock bolts: (a) intact rock bolt and (b) rock bolt with grout loss.

effect of defect size on the attenuation and behavior of wave echo in this study. For brevity, only eight distinguishable waveforms of nodal displacement on the loading surface are plotted in Figure 11. As discussed in Figure 11, the wave groups highlighted in the first red and green boxes are identified as defect echoes, whereas the end echoes are presented in the second red box of mode $L(0,3)$ and green box of mode $L(0,1)$.

The propagation of waveforms in Figure 11 shows that the wave echoes are apparently affected by the defect size. As the defect size increases, the amplitude of defect echoes of mode $L(0,1)$ indicated in the first green box rises much more than the amplitude of defect echoes of mode $L(0,3)$ presented in the first red box. Moreover, the end wave echo of mode $L(0,1)$ in the second green box decreases significantly compared to the echo of mode $L(0,3)$. The defect echo of mode $L(0,1)$ is more suitable for use in sensitivity analysis to indicate defects. The results also confirm that the interpretation of waveforms in rock bolts based on modes identification is effective, and the sensitivity of the mode $L(0,1)$ to the defect is not affected by the defect size.

In order to improve the precision, a mode-based reflection coefficient $R$ is proposed in this paper to evaluate the sensitivity of wave modes to the defect size. The reflection coefficient is calculated as the amplitude ratio of defect/end echoes and incident wave as follows:

$$
R=\frac{d_{i}}{P_{i}}
$$

where $i=1,2, \ldots, d_{1}$ is the peak-peak value of the defect echo of mode $L(0,1), d_{3}$ is the peak-peak value of the defect echo of mode $L(0,3), P_{1}$ is the peak-peak value of the end echo of mode $L(0,1), P_{3}$ is the peak-peak value of the end echo of mode $L(0,3)$, and $P$ is the peak-peak value of the incident wave echo.

The calculated reflection coefficients are plotted against the defect percentage of sectional area in Figures 12(a) and 12(b). The results in Figure 12(a) show that the linear relationship between the reflection coefficients $d_{1} / P$ of mode $L(0,1)$ and $d_{3} / P$ of mode $L(0,3)$ and the defect percentage of sectional area was significant. The increasing rate of coefficients $d_{1} / P$ of mode $L(0,1)$ is almost doubled compared to that of coefficient of $d_{3} / P$ of mode $L(0,3)$.
The variations of coefficients $P_{1} / P$ of mode $L(0,1)$ and $P_{3} / P$ of mode $L(0,3)$ generally followed the same trend. As the defect percentage of sectional area increased, the coefficients drop significantly when the defect percentage ranges from 0 to about $50 \%$. And then the coefficients showed a small fluctuation when the defect percentage ranges from $50 \%$ to $70 \%$. When the defect percentage ranges from $80 \%$ to $100 \%$, the defect is almost section drive-through; the coefficients showed a small rise and decline.

In Figure 12(b), the coefficient $d_{1} / P_{1}$ of mode $L(0,1)$ increases markedly to about 1.86 at the defect percentage of $57.19 \%$, following by a gentle increase up to a defect of $78.63 \%$. Finally, there is a rapid leap at a defect percentage of $95.31 \%$. The curve of $d_{3} / P_{3}$ of mode $L(0,3)$ rises gradually over the entire range of tested defect size. The coefficients $d_{1} / P_{1}$ of mode $L(0,1)$ are more sensitive to the defect percentage of section area. Although the end echoes of mode $L(0,1)$ and $L(0,3)$ show a fluctuation when the defect percentage ranges from $80 \%$ to $100 \%$ in the range in $80 \%$ to $100 \%$, the coefficient of defect echo to the end echo shows a good monotonicity, which will benefit in quantitatively detecting the loss of grout. It is found that the defect with low attenuation exists, and the amplitude depends on the size of the defect.

\section{Conclusions}

Based on the numerical method and dispersion curves of guided waves, this paper presents an approach of interpreting waveforms by characterizing each group of wave echo to the exact mode to analyze the guided waves propagation aimed at increasing the accuracy of inspection of rock bolts. It is found that the wave modes $L(0,1)$ and $L(0,3)$ at low frequency $(100 \mathrm{kHz})$ are the main modes of wave echoes. Moreover, the wave group of mode $L(0,1)$ propagates in both the steel and grout, while the wave group of mode $L(0,3)$ propagates mainly in the steel bar. Therefore, it could be considered that the mode $L(0,1)$ is more sensitive to the loss of grout. The different modes of main wave groups of the experiments conducted on an intact grouted rock bolt and a rock bolt with grout loss are identical with the numerical results identified by the approach of waveform interpretation, which verify the approach of waveform interpretation. 

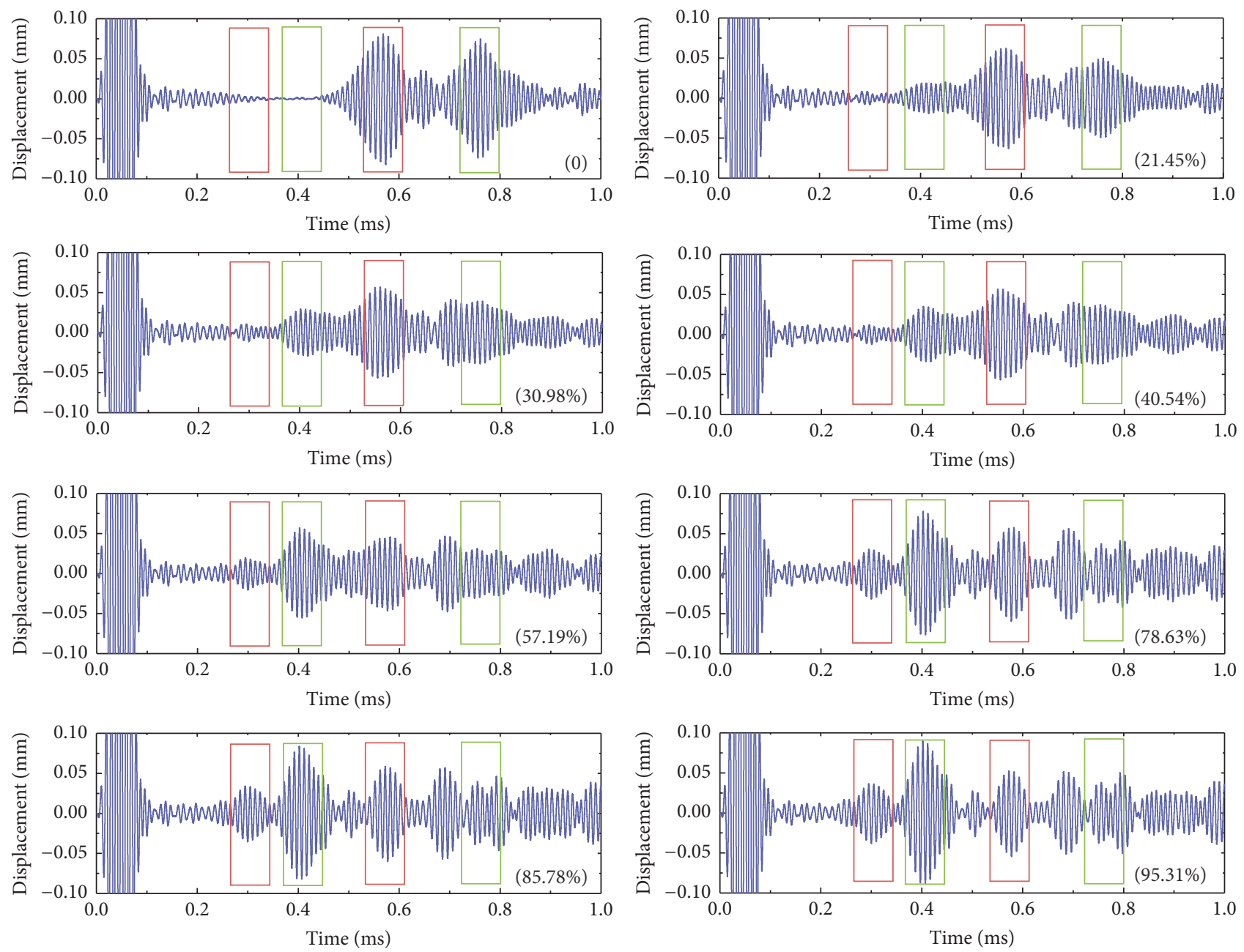

FIGURE 11: Waveforms of node displacement on loading surface of a defective rock bolt.

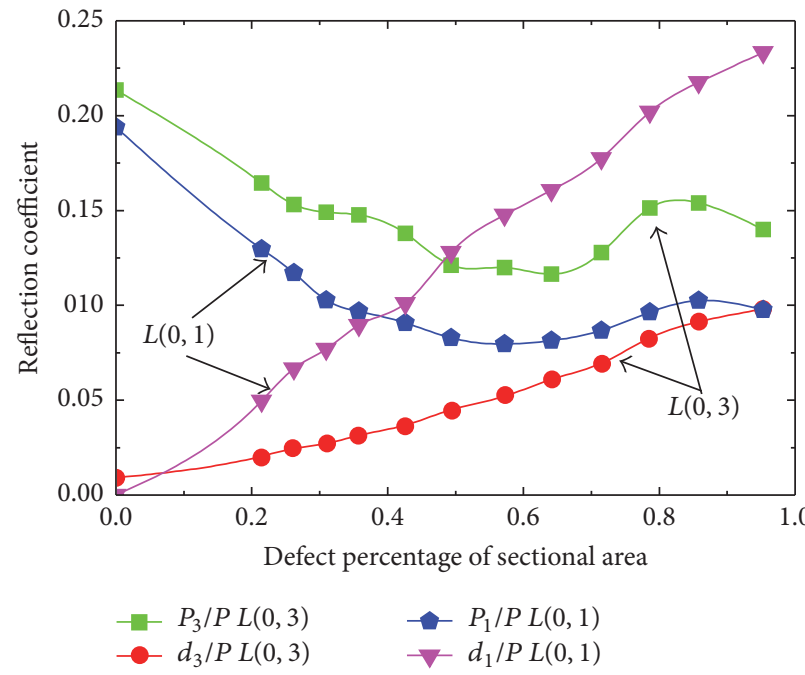

(a)

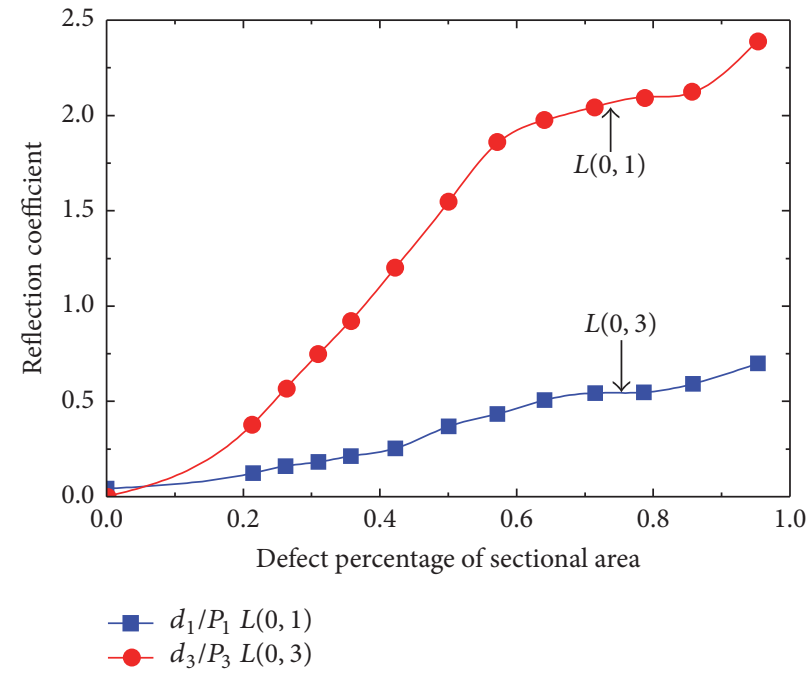

(b)

FIGURE 12: Reflection coefficient against defect percentage of dimension of sectional area. 
In addition to that, a parametric study is conducted on rock bolt models with different sectional defect size. A mode-based reflection coefficient $R$ is proposed to evaluate the sensitivity of wave modes to the defect size. The linear relationship between the reflection coefficients $d_{1} / P$ of mode $L(0,1)$ and $d_{3} / P$ of mode $L(0,3)$ and the defect percentage of sectional area is significant. Although the coefficients $P_{1} / P$ of mode $L(0,1)$ and $P_{3} / P$ of mode $L(0,3)$ shows a fluctuation when the defect percentage ranges from $80 \%$ to $100 \%$, the coefficient of $d_{1} / P_{1}$ and $d_{3} / P_{3}$ (defect echo to the end echo) shows a good monotonicity, which will benefit in quantitatively detecting the loss of grout. By contrast, the coefficients $d_{1} / P_{1}$ of mode $L(0,1)$ are more sensitive to the defect percentage of sectional area. The sensitivity of wave modes to grout defect is not affected by the defect size. The analyses also confirm the accuracy of the method of mode identification. Further investigation of wave propagation for rock bolts with different defects (e.g., geometry, length, and location) should be performed to characterize the impact of defects. Guidance on how the NDT technique can be used for detection of grout loss for rock bolts accurately should be provided.

The focus of this study is on the approach of waveform interpretation based on wave mode identification to enhance inspection sensitivity and accuracy of using NDT for rock bolt quality inspection. The analysis outcomes presented in this study are for the specific type of grout defect under consideration. It should be noted that the actual situation is more complicated due to various influencing factors, including corrosion defect, bonding defect, concrete stress, soil stress, ground water, and soil type. More types of defects should be investigated in the future theoretical and experimental work.

\section{Appendix}

$$
\begin{aligned}
& \mathbf{M}=\left[\begin{array}{llllll}
a_{11} & a_{12} & a_{13} & a_{14} & -a_{15} & -a_{16} \\
a_{21} & a_{22} & a_{23} & a_{24} & -a_{25} & -a_{26} \\
a_{31} & a_{32} & a_{33} & a_{34} & -a_{35} & -a_{36} \\
a_{41} & a_{42} & a_{43} & a_{44} & -a_{45} & -a_{46} \\
a_{51} & a_{52} & a_{53} & a_{54} & -a_{55} & -a_{56} \\
a_{61} & a_{62} & a_{63} & a_{64} & -a_{65} & -a_{66}
\end{array}\right], \\
& a_{11}=\left\{\frac{2 \mu_{1} \alpha_{1}}{R_{2}} J_{1}\left(\alpha_{1} R_{2}\right)\right. \\
& \left.-\left[\left(2 \mu_{1}+\lambda_{1}\right) \alpha_{1}^{2}+\lambda_{1} k^{2}\right] J_{0}\left(\alpha_{1} R_{2}\right)\right\} \text {, } \\
& a_{12}=\left\{\frac{2 \mu_{1} \alpha_{1}}{R_{2}} Y_{1}\left(\alpha_{1} R_{2}\right)\right. \\
& \left.-\left[\left(2 \mu_{1}+\lambda_{1}\right) \alpha_{1}^{2}+\lambda_{1} k^{2}\right] Y_{0}\left(\alpha_{1} R_{2}\right)\right\}, \\
& a_{13}=2 \mu_{1} i k \beta_{1}^{2}\left\{\frac{1}{\beta_{1} R_{2}} J_{1}\left(\beta_{1} R_{2}\right)-J_{0}\left(\beta_{1} R_{2}\right)\right\} \text {, }
\end{aligned}
$$

$$
\begin{aligned}
& a_{14}=2 \mu_{1} i k \beta_{1}^{2}\left\{\frac{1}{\beta_{1} R_{2}} Y_{1}\left(\beta_{1} R_{2}\right)-Y_{0}\left(\beta_{1} R_{2}\right)\right\} \text {, } \\
& a_{15}=\left\{\frac{2 \mu_{2} \alpha_{2}}{R_{2}} J_{1}\left(\alpha_{2} R_{2}\right)\right. \\
& \left.-\left[\left(2 \mu_{2}+\lambda_{2}\right) \alpha_{2}^{2}+\lambda_{2} k^{2}\right] J_{0}\left(\alpha_{2} R_{2}\right)\right\} \text {, } \\
& a_{16}=2 \mu_{2} i k \beta_{2}^{2}\left\{\frac{1}{\beta_{2} R_{2}} J_{1}\left(\beta_{2} R_{2}\right)-J_{0}\left(\beta_{2} R_{2}\right)\right\} \text {, } \\
& a_{21}=\left[-2 \mu_{1} i k \alpha_{1} J_{1}\left(\alpha_{1} R_{2}\right)\right] \text {, } \\
& a_{22}=\left[-2 \mu_{1} i k \alpha_{1} Y_{1}\left(\alpha_{1} R_{2}\right)\right] \text {, } \\
& a_{23}=\left[\left(k^{2} \beta_{1}-\beta_{1}^{3}\right) \mu_{1} J_{1}\left(\beta_{1} R_{2}\right)\right] \text {, } \\
& a_{24}=\left[\left(k^{2} \beta_{1}-\beta_{1}^{3}\right) \mu_{1} Y_{1}\left(\beta_{1} R_{2}\right)\right] \text {, } \\
& a_{25}=\left[-2 \mu_{2} i k \alpha_{2} J_{1}\left(\alpha_{2} R_{2}\right)\right] \text {, } \\
& a_{26}=\left[\left(k^{2} \beta_{2}-\beta_{2}^{3}\right) \mu_{2} J_{1}\left(\beta_{2} R_{2}\right)\right] \text {, } \\
& a_{31}=\left\{\frac{2 \mu_{1} \alpha_{1}}{R_{1}} J_{1}\left(\alpha_{1} R_{1}\right)\right. \\
& \left.-\left[\left(2 \mu_{1}+\lambda_{1}\right) \alpha_{1}^{2}+\lambda_{1} k^{2}\right] J_{0}\left(\alpha_{1} R_{1}\right)\right\} \text {, } \\
& a_{32}=\left\{\frac{2 \mu_{1} \alpha_{1}}{R_{1}} Y_{1}\left(\alpha_{1} R_{1}\right)\right. \\
& \left.-\left[\left(2 \mu_{1}+\lambda_{1}\right) \alpha_{1}^{2}+\lambda_{1} k^{2}\right] Y_{0}\left(\alpha_{1} R_{1}\right)\right\} \text {, } \\
& a_{33}=2 \mu_{1} i k \beta_{1}^{2}\left\{\frac{1}{\beta_{1} R_{1}} J_{1}\left(\beta_{1} R_{1}\right)-J_{0}\left(\beta_{1} R_{1}\right)\right\} \text {, } \\
& a_{34}=2 \mu_{1} i k \beta_{1}^{2}\left\{\frac{1}{\beta_{1} R_{1}} Y_{1}\left(\beta_{1} R_{1}\right)-Y_{0}\left(\beta_{1} R_{1}\right)\right\} \text {, } \\
& a_{35}=0 \text {, } \\
& a_{36}=0 \text {, } \\
& a_{41}=\left[-2 \mu_{1} i k \alpha_{1} J_{1}\left(\alpha_{1} R_{1}\right)\right] \text {, } \\
& a_{42}=\left[-2 \mu_{1} i k \alpha_{1} Y_{1}\left(\alpha_{1} R_{1}\right)\right] \text {, } \\
& a_{43}=\left[\left(k^{2} \beta_{1}-\beta_{1}^{3}\right) \mu_{1} J_{1}\left(\beta_{1} R_{1}\right)\right] \text {, } \\
& a_{44}=\left[\left(k^{2} \beta_{1}-\beta_{1}^{3}\right) \mu_{1} Y_{1}\left(\beta_{1} R_{1}\right)\right] \text {, } \\
& a_{45}=0 \text {, } \\
& a_{46}=0 \text {, } \\
& a_{51}=-\alpha_{1} J_{1}\left(\alpha_{1} R_{2}\right) \text {, } \\
& a_{52}=-\alpha_{1} Y_{1}\left(\alpha_{1} R_{2}\right) \text {, } \\
& a_{53}=-i k \beta_{1} J_{1}\left(\beta_{1} R_{2}\right) \text {, }
\end{aligned}
$$




$$
\begin{aligned}
& a_{54}=-i k \beta_{1} Y_{1}\left(\beta_{1} R_{2}\right), \\
& a_{55}=-\alpha_{2} J_{1}\left(\alpha_{2} R_{2}\right), \\
& a_{56}=-i k \beta_{2} J_{1}\left(\beta_{2} R_{2}\right), \\
& a_{61}=i k J_{0}\left(\alpha_{1} R_{2}\right), \\
& a_{62}=i k Y_{0}\left(\alpha_{1} R_{2}\right), \\
& a_{63}=\beta_{1}^{2} J_{0}\left(\beta_{1} R_{2}\right), \\
& a_{64}=\beta_{1}^{2} Y_{0}\left(\beta_{1} R_{2}\right), \\
& a_{65}=i k J_{0}\left(\alpha_{2} R_{2}\right), \\
& a_{66}=\beta_{2}^{2} J_{0}\left(\beta_{2} R_{2}\right) .
\end{aligned}
$$

\section{Competing Interests}

The authors declare that there is no conflict of interests regarding the publication of this article.

\section{Acknowledgments}

This work was funded by the Natural Sciences and Engineering Research Council of Canada (NSERC), Ontario Centers of Excellences (OCE), DYWIDAG-Systems International Canada Ltd., National Basic Research Program of China (no. 2013CB227902), and Natural Science Foundation of China (Grant no. 51574059). The authors also want to acknowledge the scholarship awarded to first author by the China Scholarship Council during her visit at Ryerson University.

\section{References}

[1] H. F. Thurner, I. Jasarevic, Z. Tavas, I. Muhovec, B. Nesrsta, and N. Krauland, "Detection of invisible faults on rockbolts in-situ," in Proceedings of the International Symposium on Rock Bolting Theory and Application in Mining and Underground Construction, August-September 1983.

[2] P. W. Jayawickrama, Y. Tinkey, G. Jie, and J. Turner, Nondestructive evaluation of installed soil nails [M.S. thesis], Texas Tech University, Lubbock, Tex, USA, 2007.

[3] S.-T. Liao, C.-K. Huang, and C.-Y. Wang, "Sonic echo and impulse response tests for length evaluation of soil nails in various bonding mediums," Canadian Geotechnical Journal, vol. 45, no. 7, pp. 1025-1035, 2008.

[4] R. Cheung and D. Lo, "Use of time-domain reflectometry for quality control of soil-nailing works," Journal Geotechnical Geoenvironmental Engineering, vol. 137, no. 12, pp. 1222-1235, 2011.

[5] M. D. Beard and M. J. S. Lowe, "Non-destructive testing of rock bolts using guided ultrasonic waves," International Journal of Rock Mechanics and Mining Sciences, vol. 40, no. 4, pp. 527-536, 2003.

[6] Y. Cui and D. H. Zou, "Assessing the effects of insufficient rebar and missing grout in grouted rock bolts using guided ultrasonic waves," Journal of Applied Geophysics, vol. 79, pp. 64-70, 2012.
[7] V. Madenga, In application of guided ultrasonic waves to grout quality testing of rock bolts [M.S. thesis], Dalhousie University, 2004.

[8] D. H. Zou, Y. Cui, V. Madenga, and C. Zhang, "Effects of frequency and grouted length on the behavior of guided ultrasonic waves in rock bolts," International Journal of Rock Mechanics and Mining Sciences, vol. 44, no. 6, pp. 813-819, 2007.

[9] D. H. S. Zou, J. Cheng, R. Yue, and X. Sun, "Grout quality and its impact on guided ultrasonic waves in grouted rock bolts," Journal of Applied Geophysics, vol. 72, no. 2, pp. 102-106, 2010.

[10] A. Demma, The Interaction of Guided Waves with Discontinuities in Structures, Imperial College London Press, London, UK, 2003.

[11] J. L. Rose, Ultrasonic Waves in Solid Media, Cambridge University Press, Cambridge, UK, 1999.

[12] V. Madenga, D. H. Zou, and C. Zhang, "Effects of curing time and frequency on ultrasonic wave velocity in grouted rock bolts," Journal of Applied Geophysics, vol. 59, no. 1, pp. 79-87, 2006.

[13] J. L. Rose, "Dispersion curves in guided wave testing," Materials Evaluation, vol. 61, no. 1, pp. 20-22, 2003.

[14] B. L. Ervin and H. Reis, "Longitudinal guided waves for monitoring corrosion in reinforced mortar," Measurement Science and Technology, vol. 19, no. 5, pp. 120-125, 2008.

[15] B. L. Ervin, D. A. Kuchma, J. T. Bernhard, and H. Reis, "Monitoring corrosion of rebar embedded in mortar using high-frequency guided ultrasonic waves," Journal of Engineering Mechanics, vol. 135, no. 1, pp. 9-19, 2009.

[16] S. Sharma and A. Mukherjee, "Monitoring corrosion in oxide and chloride environments using ultrasonic guided waves," Journal of Materials in Civil Engineering, vol. 23, no. 2, pp. 207211, 2011.

[17] S. Sharma and A. Mukherjee, "Nondestructive evaluation of corrosion in varying environments using guided waves," Research in Nondestructive Evaluation, vol. 24, no. 2, pp. 63-88, 2013.

[18] J. S. Whittier and J. P. Jones, "Axially symmetric wave propagation in a two-layered cylinder," International Journal of Solids and Structures, vol. 3, no. 4, pp. 657-675, 1967.

[19] M. J. S. Lowe, D. N. Alleyne, and P. Cawley, "Defect detection in pipes using guided waves," Ultrasonics, vol. 36, no. 1-5, pp. 147-154, 1998.

[20] F. Moser, L. J. Jacobs, and J. Qu, "Modeling elastic wave propagation in waveguides with the finite element method," NDT \& E International, vol. 32, no. 4, pp. 225-234, 1999.

[21] L. Maio, V. Memmolo, F. Ricci, N. D. Boffa, E. Monaco, and R. Pecora, "Ultrasonic wave propagation in composite laminates by numerical simulation," Composite Structures, vol.121, pp. 6474, 2015.

[22] M. Redwood, "Mechanical waveguides: the propagation of acoustic and ultrasonic waves in fluids and solids with boundaries," in Mechanical Waveguides: The Propagation of Acoustic and Ultrasonic Waves in Fluids and Solids with Boundaries, pp. 66-71, Pergamon Press, London, UK, 1960.

[23] F. Seco and A. R. Jiménez, Modelling the Generation and Propagation of Ultrasonic Signals in Cylindrical Waveguides, InTech, Rijeka, Croatia, 2012.

[24] J. Achenbach, Wave Propagation in Elastic Solids, Elsevier Press, New York, NY, USA, 2012. 


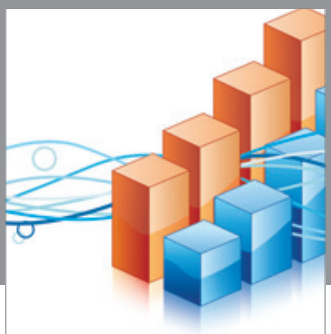

Advances in

Operations Research

vatem alat4

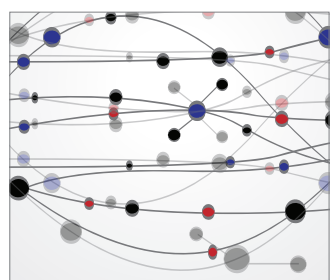

\section{The Scientific} World Journal
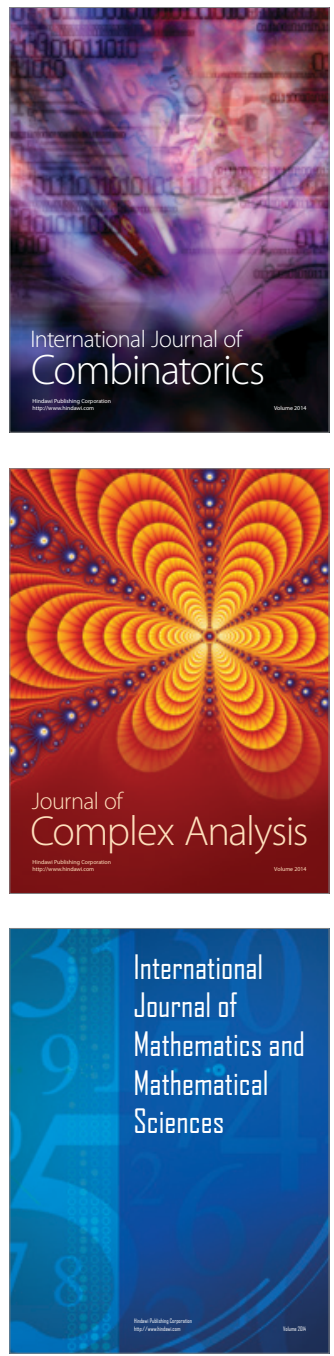
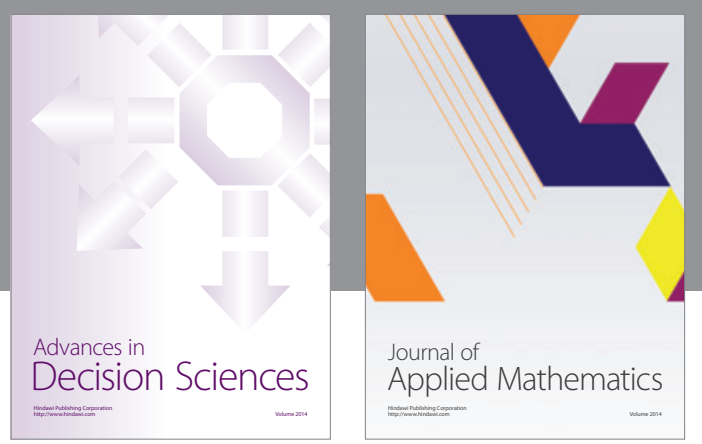

Algebra

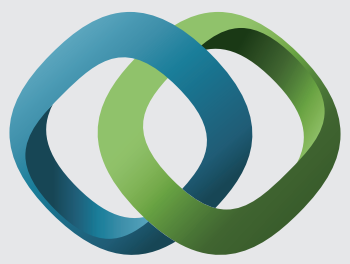

\section{Hindawi}

Submit your manuscripts at

https://www.hindawi.com
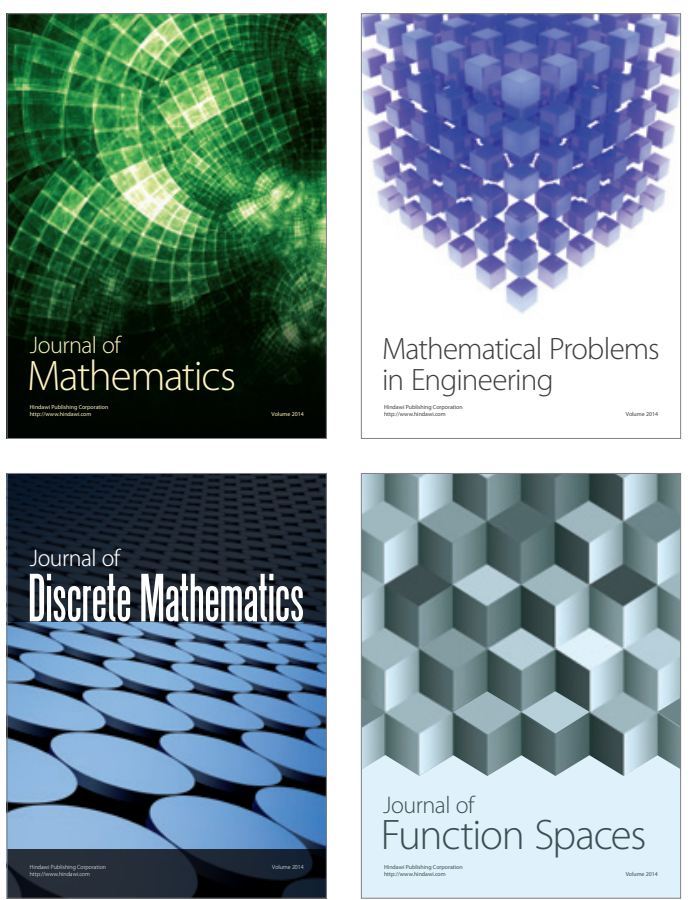

Mathematical Problems in Engineering
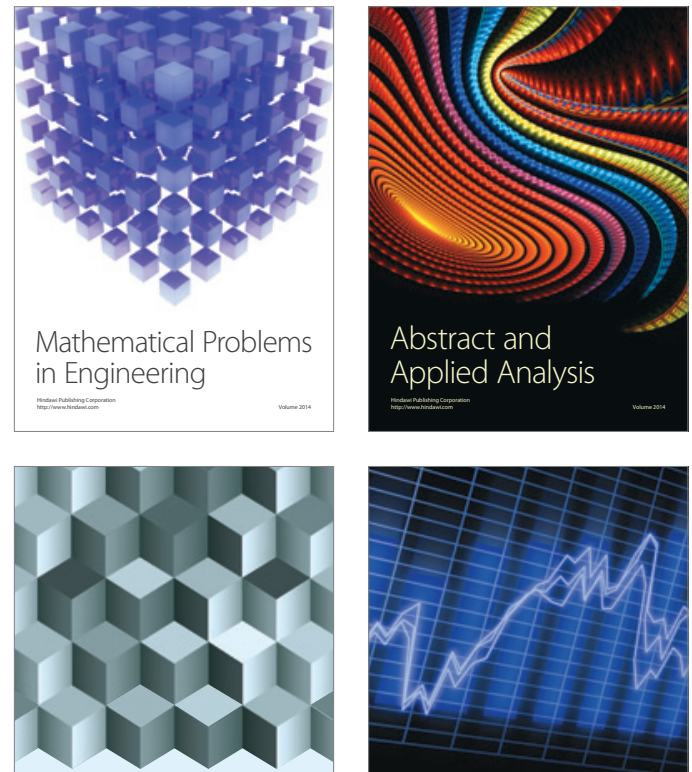

Journal of

Function Spaces

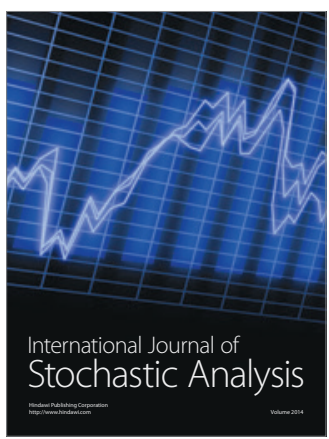

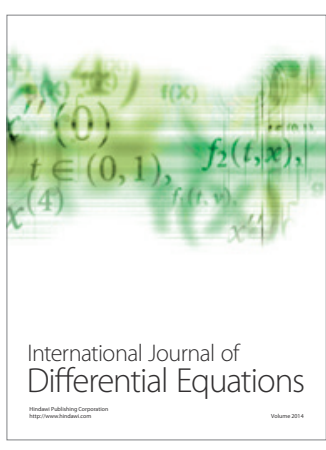
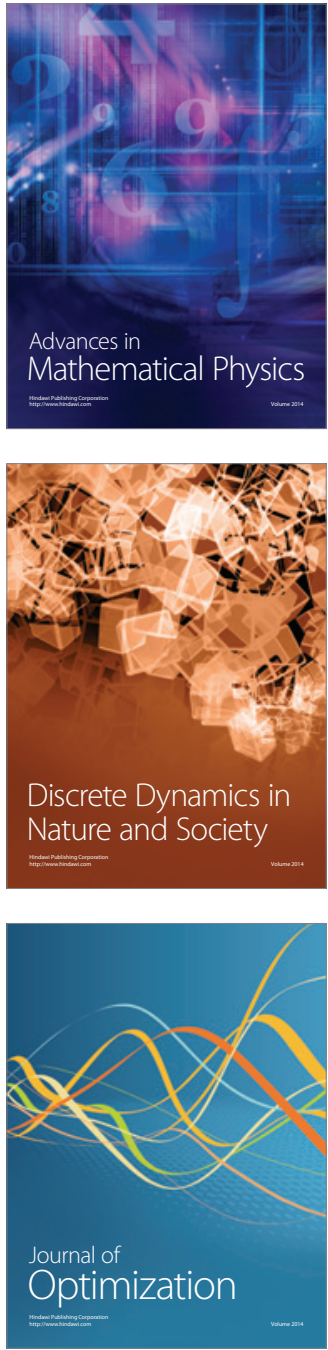